\title{
Secreted HMGB1 from Wnt activated intestinal cells is required to maintain a crypt progenitor phenotype
}

\author{
Karen R. Reed ${ }^{1}$, Fei Song ${ }^{2,3}$, Maddy A. Young ${ }^{1}$, Nurudeen Hassan ${ }^{1,4}$, Daniel $\mathrm{J}$. \\ Antoine $^{3}$, Nesibe-Princess B. Gemici ${ }^{1}$, Alan R. Clarke ${ }^{1}$, John R. Jenkins ${ }^{3}$ \\ ${ }^{1}$ European Cancer Stem Cell Research Institute, School of Biosciences, Cardiff University, Cardiff, CF24 4HQ, UK \\ ${ }^{2}$ Infrafrontier GmbH, Neuherberg / München, 85764, Germany \\ ${ }^{3}$ Institute of Translational Medicine, University of Liverpool, Liverpool, L69 3BX, UK \\ ${ }^{4}$ Cardiff School of Health Sciences at Cardiff Metropolitan University, Cardiff, CF5 2YB, UK \\ Correspondence to: Karen R. Reed, email: ReedKR@Cardiff.ac.uk \\ Keywords: Hmgb 1, colorectal cancer, intestinal stem cells, Apc, Wnt signalling \\ Received: March 22, $2016 \quad$ Accepted: May 29, $2016 \quad$ Published: June 15, 2016
}

This is an open access article first published in Oncotarget under the terms of the Creative Commons Attribution License.

\section{ABSTRACT}

Background and Aims: Colorectal cancer (CRC) arises via multiple genetic changes. Mutation of the tumour suppressor gene APC, a key regulator of Wnt signalling, is recognised as a frequent early driving mutation in CRC. We have previously shown that conditional loss of $A p C$ within the murine small intestine (Apcfoxmice) results in acute Wnt signalling activation, altered crypt-villus architecture and many hallmarks of neoplasia. Our transctipomic profiling (Affymetrix Microarrays) and proteomic profiling (iTRAQ-QSTAR) of Apc-deficient intestine inferred the involvement of High Mobility Group Box 1 (Hmgb1) in CRC pathogenesis. Here we assess the contribution of HMGB1 to the crypt progenitor phenotype seen following Apc loss.

Results: Elevated HMGB1 was confirmed in intestinal epithelia and serum following conditional loss of Apc. Treatment of Apc flox mice with anti-HMGB1 neutralising antibody significantly reduced many of the crypt progenitor phenotypes associated with Apc loss; proliferation and apoptosis levels were reduced, cell differentiation was restored and the expansion of stem cell marker expression was eradicated.

Methods: Hmgb1 levels in intestinal epithelia and serum in $A p c^{\text {flox }}$ and $A p c^{\text {Min }}$ mice were assessed using qRT-PCR, Western blot and ELISA assays. The functional importance of elevated extracellular Hmgb1 was assessed using an anti-HMGB1 neutralising antibody in Apc flox mice.

Conclusions: HMGB1 is expressed and secreted from intestinal epithelial cells in response to Wnt signalling activation. This secreted HMGB1 is required to maintain nearly all aspects of the crypt progenitor phenotype observed following Apc loss and add to the body of accumulating evidence indicating that targeting HMGB1 may be a viable novel therapeutic approach.

\section{INTRODUCTION}

Wnt signalling is a key pathway which regulates normal intestinal homeostasis [1]. It is widely recognised that deregulation of the Wnt pathway underpins the early stages of colorectal cancer $[2,3]$. Genetically modified mouse models in which the key Wnt regulator and tumour suppressor gene Adenomatous polyposis coli $(A p c)$ has been rendered inactive, have greatly assisted in identifying factors that contribute to the pathogenesis of CRC (reviewed [4]). We have previously characterised the murine intestine following the conditional deletion of $A p c$, and shown a rapid perturbation of differentiation, migration, proliferation, and apoptosis accompanying the acute activation of Wnt signalling, such that the Apc-deficient cells maintain a "crypt progenitorlike" phenotype and many hallmarks of neoplasia [5]. Furthermore, we have previously conducted 
Transcriptomic Affymetrix microarray analysis $[5,6]$ and proteomic profiling using iTRAQ-QSTAR [7] to identify genes that are mis-regulated at these early stages of neoplasia following the loss of $A p c$, and thereby identify factors that potentially contribute to CRC pathogenesis. These analyses have inferred the involvement of the High Mobility Group Box 1 (Hmgbl) gene.

HMGB1 is an abundant multifunctional protein possessing diverse biological activities in normal cells, and has been the subject of intense investigation in recent cancer research being implicated in tumour formation, progression, metastasis and response to chemotherapeutics (reviewed [8]). Within the nucleus, HMGB1 functions to regulate transcription, replication, DNA repair and genomic stability [9-12], while cytoplasmic or cellmembrane bound HMGB1 can regulate cell motility [1315]. Furthermore, HMGB1 can be released by cells, both passively and actively, whereupon it can act as a cytokine and damage associated molecular pattern (DAMP) molecule [16].

HMGB1 is passively released from cells that have died in a traumatic, non-programmed way (necrosis or pyroptosis) $[17,18]$. Significantly, apoptotic cells modify their chromatin so as to bind HMGB1, preventing its release during this form of programmed cell death [17]. Several cell types (including activated immune cells; monocytes, macrophages and natural killer cells) have the ability to actively secrete HMGB1, via a dedicated pathway, and thus produce a damage signal without associated cell death [19]. Once released, extracellular HMGB1 can interact with a number of different receptors including TLR2, TLR4 and RAGE to elicit different responses (reviewed [16, 20]). Post-translational modifications of HMGB1, particularly in relation to the redox state of three cysteines, can influence this receptor binding [20]. The all-thiol form of HMGB1 (in which the cysteines are reduced) is the predominant intra-cellular form and it is this form that is passively released following tissue damage. Once released, all-thiol HMGB1 form elicits the chemo-attractant activities of HMGB1, via the CXCR4 receptor, but this form is not capable of inducing cytokine production [21]. Conversely, disulphideHMGB1 promotes activation of the NF- $\kappa B$ pathway and proinflammatory cytokine production in fibroblasts and macrophages [21]. Both the all-thiol and disulphide forms of HMGB1 are actively secreted by immune cells, but oxidation of the extra-cellular all-thiol form by reactive oxygen species (ROS) results in accumulation of disulphide and oxidised forms of HMGB1 [21].

Elevated levels of HMGB1 have been reported for numerous cancers, including CRC, and higher levels of HMGB1 has been show to positively correlate with tumour invasion and metastasis (both distant and lymph-node), and reduced survival for CRC patients [22, 23]. Indeed, 7 out of 14 datasets in the Oncomine database display a $>2$ fold up-regulated of HMGB1 $(p<0.05)$ for the cancer $\mathrm{v}$ normal comparison for colorectal cancer, while, one of the four cohorts detailed on Prognoscan (contributed by Staub) displays a statistical significant association between the levels of HMGB1 and clinical outcome (high levels of HMGB1 being associated with a poor prognosis. Both databases accessed 25/5/16). Moreover, elevated levels of serum HMGB1 are associated with the onset of inflammation following the administration of dextran sulfate sodium salt (DSS) in a mouse model of colitis-associated cancer (DSS-treated $A p c^{M i n}$ mice) [24]. Administration of a neutralizing anti-HMGB1 antibody in this mouse model markedly reduced the level of intestinal inflammation and decreased tumour incidence and size, thereby highlighting the potential usefulness of HMGB1 as a target for the treatment of colitis and the prevention of colitis-associated cancer [24]. Furthermore, loss of the RAGE receptor significantly impacts on intestinal tumourigenesis in $A p c^{\text {Min }}$ mice [25].

Here we demonstrate that elevated levels of HMGB1 occur within the intestine and the serum, following the conditional deletion of $A p c$ and acute activation of Wnt signalling in the mouse intestine (using $A p c^{f l o x}$ mice). We further show that treatment of $A p c^{f f o x}$ mice with a neutralizing anti-HMGB1 antibody restores many of the aberrant features associated with loss of $A p c$ toward normal. The exact mechanisms by which HMGB1 regulates Wnt signalling and intestinal stem cells remains to be determined, however, our work adds to the accumulating evidence implicating HMGB1 has potential for cancer therapy.

\section{RESULTS}

\section{Wnt signalling activation results in up-regulated Hmgb1 expression}

Apc is a known key regulator of Wnt signalling, and critically important in regulating normal intestinal homeostasis. Cre-LoxP driven recombination of $A p c$ within the mouse intestine using an Ah-Cre recombinase to drive recombination of LoxP flanked $A p c$ alleles, has previously been shown to result in acute activation of Wnt signalling and many hallmarks of neoplasia, including increased proliferation and apoptosis and loss of differentiation and migration [5]. Our previous Affymetrix microarray analysis $[5,6]$ and proteomic profiling using iTRAQ-QSTAR [7] analysis inferred the involvement of High Mobility Group Box 1 (Hmgbl). Subsequently, upregulation of $\mathrm{Hmgbl}$ expression and elevation of HMGB1 within the intestinal epithelia was confirmed and shown to occur concomitantly with nuclear accumulation of $\beta$-catenin (and hence Wnt signalling activation) following the conditional loss of $A p c$ (Figure 1). It is of interest to note that a Tcf binding element (TBE sequence $[\mathrm{at}][\mathrm{at}] \mathrm{CAA}[\mathrm{at}] \mathrm{G})$ can be found within the human HMGB1 promoter [26], which combined with our data indicates Hmgbl is likely a Wnt target gene. 
Different post-translational modifications of HMGB1 are known to be associated with different modes of release, and extra-cellular functions [21, 27]. ELISA analysis confirmed the significant elevation of HMGB1 levels in mouse serum day 5 post induction of $A p c$ loss (Figure 2A), although the cellular source for this secreted HMGB1 is not known. These levels are similar to those seen in aged (approximately 6 months old) $A p c^{\text {Min }}$ mice possessing an intestinal tumour burden, but due to a greater variability in aged $A p c^{M i n}$ mice, significant elevation in these samples could not be proven (Figure 2A). To further characterise the form of the secreted
HMGB1, tandem mass spectrometry (MS/MS) analysis from $\mathrm{Apc}^{+/+}$(control) and $A p c^{A / f l}$ serum, Day 4 post induction was undertaken (Figure 2B). This demonstrated the acquisition of hyper-acetylated and methylated forms of HMGB1 following Apc loss, both of which are forms that are associated with release due to inflammasome activation, pyroptosis and leukocyte and neutrophile derived HMGB1 (reviewed [27]). Thus, activation of Wnt signalling within the intestinal epithelia leads to the active secretion of HMGB1, and significantly higher levels of HMGB1 in the serum.

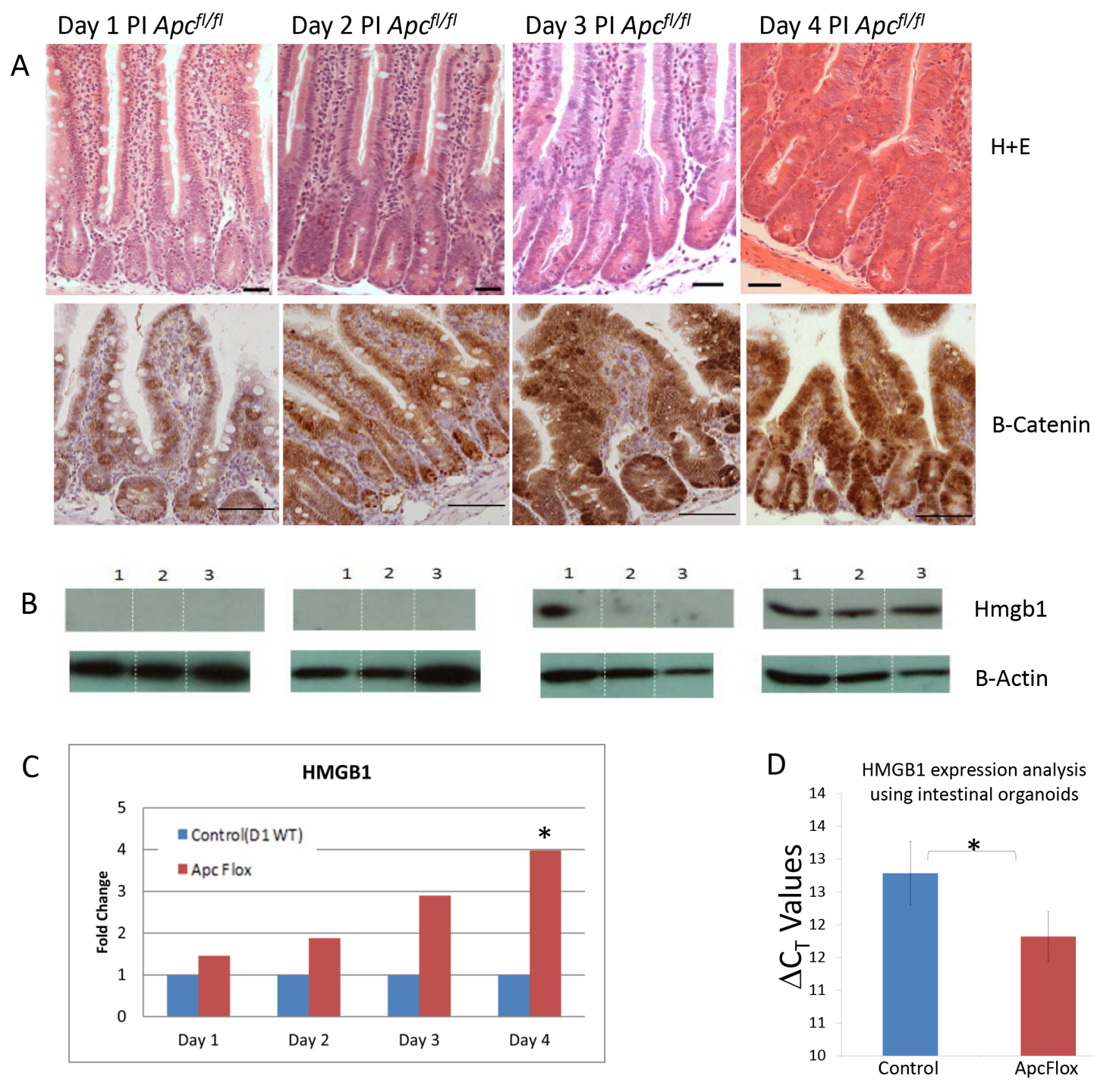

Figure 1: Accumulation of intestinal Hmgb1 following the loss of Apc. A. H+E stained intestine and $\beta$-catenin IHC following the conditional loss of $A p c$. Days post induction are indicated at the top. Elevated nuclear accumulation of $\beta$-catenin occurs from day 3 PI onward. B. Western Blot analysis of HMGB1 levels on triplicate epithelia cell extracts from each time point. Elevation of HMGB1 was first seen at day 3 PI. C. qRT-PCR of Hmbgl expression levels presented as relative fold change within epithelia cell extracts compared to day 1 WT demonstrates a significant (*) induction 4 days PI (determined using Whitney $\mathrm{U}$ test of $\Delta C T$ values at P $<0.05$ ). D. qRT-PCR of Hmbgl expression levels presented as $\Delta \mathrm{CT}$ values from control (wildtype) and Apc deficient intestinal organoid cultures. The values shown represent a 1.9 fold over-expression of Hmgb1 in the Apc deficient organoids. 


\section{Neutralising anti-HMGB1 treatment mitigates the $A p c^{f l o x}$ phenotype and reduces pro- inflammatory signals}

The functional significance of the elevated extracellular HMGB1 levels seen following intestinal Wnt signalling activation was assessed by treating induced $A p c^{f o x}$ mice with neutralising anti-HMGB1 antibody for two days prior to sample collection. Although this treatment regime did not alter the levels of HMGB1 within intestinal epithelial cell extracts, the levels of total HMGB1 seen within the whole intestine was significantly reduced (Figure 3A). Characterisation of the intestinal histology revealed that the extent of the "floxed" region associated with the loss of $A p c$ was significantly reduced following neutralising anti-HMGB1 antibody treatment (Figure 3B). Furthermore, microscopic scoring and Immunohistochemical (IHC) analysis clearly showed a significant reduction in the level of epithelia cell proliferation (Figure 3C, 3D) and apoptosis (Figure $3 \mathrm{E})$ within the "floxed" region, while the activation of stem cell markers was significantly reduced (Figure 3F) and goblet cell differentiation was restored to normal (Supplementary Figure 1). Thus, the extra-cellular levels of HMGB1 positively contribute to the phenotype observed following the loss of $A p c$ within the intestinal epithelia. qRT-PCR analysis has shown a significant reduction in the HMGB1 receptor RAGE (1.8 fold down), in the whole intestinal tissue from $A p c^{f l o x}$ treated with neutralising anti-HMGB1 antibody compared to those treated with control IgY (Figure 4). The importance of the HMGB1-RAGE signalling pathway in initiation of intestinal tumourigenesis has previously been described and a positive feedback loop between ligand and receptor signalling proposed [25]. Our results corroborate these findings, showing reduction in the levels of the ligand HMGB1, results in the reduction in the levels of RAGE receptor, and an overall normalisation of the Wnt signalling induced "crypt-Progenitor like" phenotype. Furthermore, qRT-PCR analysis using whole intestinal tissue has shown a significant reduction in the proinflammatory marker and Wnt signalling target gene CD44 (2.7 fold down) and cytokine Il6 (10.5 fold down) in the $A p c^{f o x}$ treated with neutralising anti-HMGB1 antibody compared to those treated with control IgY (Figure 4). However, generic alteration of Wnt signalling target genes was not seen; levels of $c M Y C$, PPARdelta, Tcf7, Lef1, Fgf4, MMP7 and Wispl were not affected by the neutralising anti-HMGB1 antibody in the $A p c^{f t o x}$ intestine.

\section{DISCUSSION}

Here we show that activation of Wnt signalling, a common and often early event in intestinal tumourigenesis, results in the up-regulation and active secretion of Hmgb1 within the intestine, which in turn results in elevated levels of serum Hmgb1. It is likely that release of HMGB1 signals via the RAGE receptor and promotes inflammation activation, which in turn feeds-back to the mechanisms regulating normal intestinal homeostasis and the stem cell

A

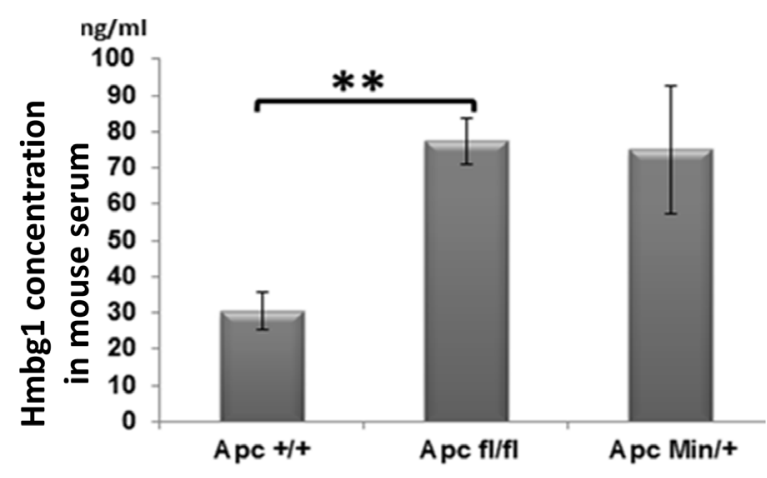

B

\begin{tabular}{|c|c|c|c|c|c|c|c|c|c|}
\hline $\begin{array}{c}\text { Mouse } \\
\text { Strain }\end{array}$ & \multicolumn{3}{|c|}{ Redox state } & \multicolumn{2}{c|}{ Hyper-Acetylation } & \multicolumn{2}{c|}{ Hypo-Acetylation } & $\begin{array}{c}\text { Mono- } \\
\text { Methyl K42 }\end{array}$ & $\begin{array}{c}\text { Unmodified } \\
\text { K42 }\end{array}$ \\
\cline { 2 - 8 } & Sulphonate & $\begin{array}{c}\text { Disulphide } \\
\text { HMGB1 }\end{array}$ & $\begin{array}{c}\text { All thiol } \\
\text { HMGB1 }\end{array}$ & NLS1 & NLS2 & NLS1 & NLS2 & & \\
\hline Apc ${ }^{+/+}$ & & & $3 / 3$ & & & $3 / 3$ & $3 / 3$ & & $3 / 3$ \\
\hline Apc $^{\mathrm{fl} / f+}$ & & $2 / 3$ & $3 / 3$ & $3 / 3$ & $3 / 3$ & $3 / 3$ & $3 / 3$ & $2 / 3$ & \\
\hline
\end{tabular}

Figure 2: Elevation of serum HMGB1 following the loss of Apc. A. ELISA analysis demonstrating significant elevation of HMGB1 serum levels in $A p c^{f o x}$ mice and increased levels in $A p c^{M i n}$ mice compared to aged match mice. B. Table showing the number of samples in which the different post-translational forms of HMGB1 were detected in the serum of $A p c^{f o x}$ mice day 4 PI, using MS/MS analysis. Empty boxes indicate this form was not detected in any of the samples analysed $(n=3)$. 
compartment within the intestinal epithelia. The process of inflammation driven intestinal repair is known to promote a tumour-promoting environment [24, 28], and the release of HMGB1 contributes to tumourigenesis in mouse models of colitis-associated cancer [24, 25, 29].

However, we have also shown that elevated levels of Hmgb1 contribute towards many of the abnormal "crypt progenitor" phenotypes that occur following the loss of $A p c$, including the expansion of intestinal stem cell marker expression. The exact mechanisms by which Hmgbl influences the intestinal stem cells remains to be elucidated, but the lack of generic alteration of Wnt signalling target genes following the neutralising antibody treatment despite the fact that HMGB1 has also been shown to be a "non-core regulator" and strong inducer of Wnt/TCF-dependent transcription [30], suggests altered Wnt signaling is not the likely underlying mechanism. Rather the influence of Hmgb1 on immune cells and the inflammatory response could be a contributing factor. However, our work provides evidence that Hmgbl is a potential Wnt signalling target gene and contributes to the body of evidence implicating Hmgb1 in cancer formation with the potential for exploitation as a therapeutic target.

B

A

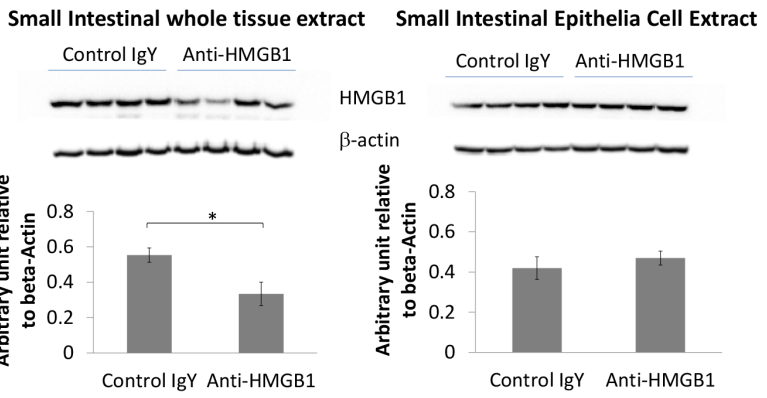

C

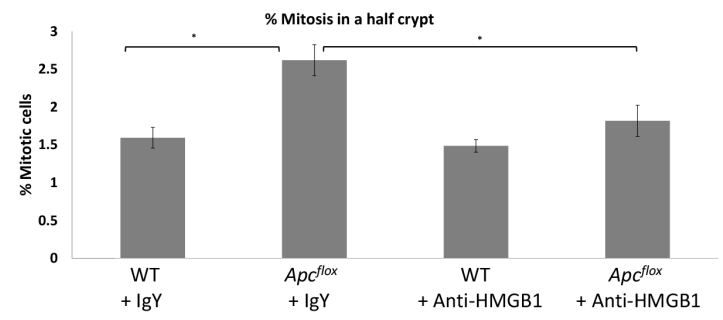

D

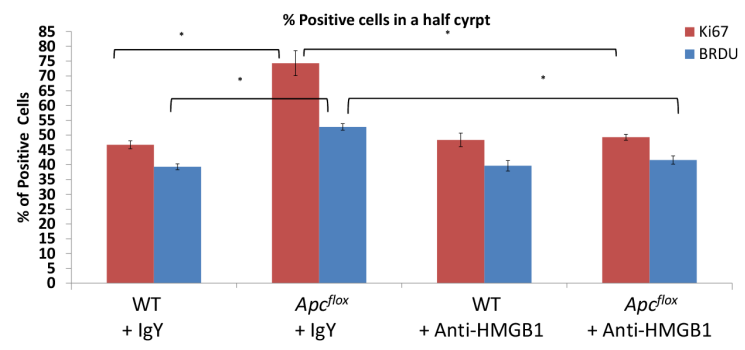

$E$

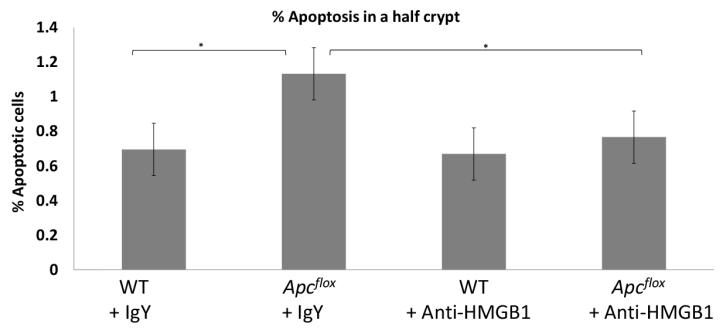

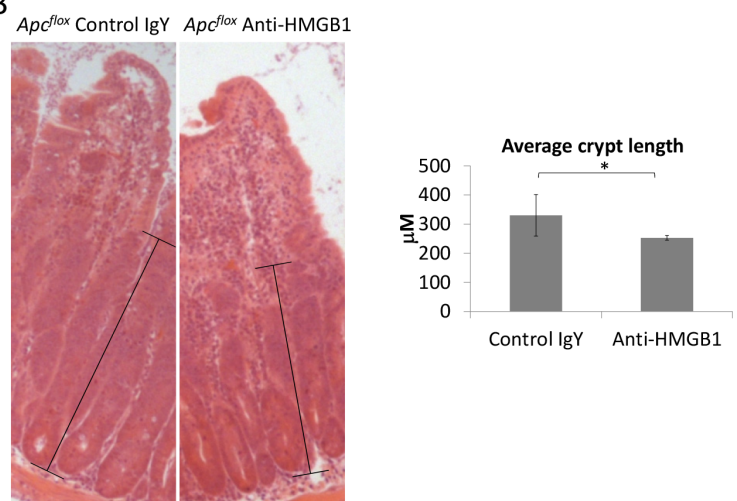

F
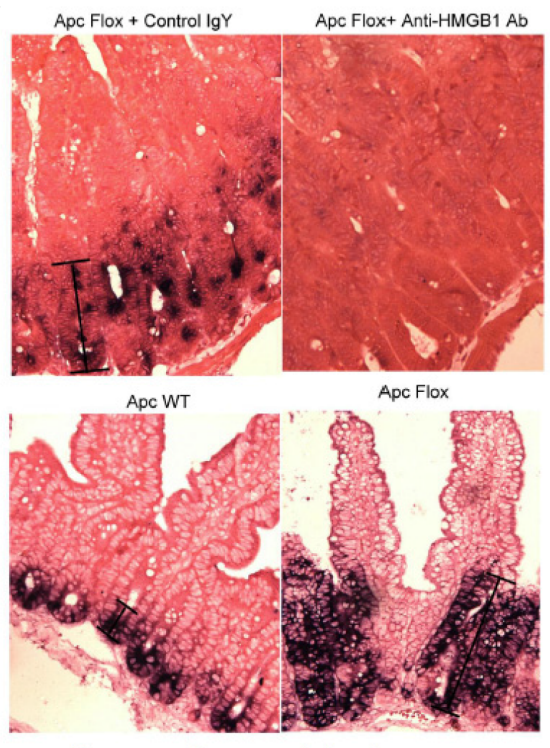

Olfm4-specific in situ hybridisation

Figure 3: Characterisation of Wnt activated intestine following neutralising HMGB1-antibody treatment. A. Western blot analysis for total HMGB1 levels in the whole intestine and epithelial cell extracts. Densitometry analysis shows a significant (40\%) reduction of HMGB1 protein level in the small intestine tissue, but no change in the epithelia cell extracts small intestine epithelial cells. B. The length from the base of a crypt to the top of the aberrant morphology displaying crypt-like features was significantly shorter following the treatment with neutralising HMGB1-antibody. C. Percent of mitotic bodies within the "floxed" region, D. percent of BRDU and Ki67 positive cells within the "floxed" region, and E. percent of apoptotic bodies within the "floxed" region are all significantly reduced following neutralising HMGB1-antibody treatment. F. Olfm 4 In situ Hybridisation shows a marked reduction within the "floxed" region in $A p c^{f o x}$ mice following neutralising HMGB1-antibody treatment. In all cases * denotes $\mathrm{p}<0.05, \mathrm{M}-\mathrm{W} \mathrm{U}$ test. 


\section{MATERIALS AND METHODS}

\section{Mice and sample preparation}

This study received ethical approval from Cardiff University's Animal Welfare and Ethical Review Body (previously known as the ERP), and all animal procedures were conducted in accordance with UK Home Office regulations. $\mathrm{AhCre}^{+} \mathrm{Apc}^{+/+}$(control) and $\mathrm{AhCre}{ }^{+} \mathrm{Apc}^{A / f /}$ mice were generated and maintained on an outbred background as previously described [5]. Cre-recombinase activity was induced from the Ah-Cre transgene by three intraperitoneal (IP) injections of $80 \mathrm{mg} / \mathrm{kg} \beta$-naphthoflavone within $24 \mathrm{~h}$ on day 0 . Mice were sacrificed, and samples collected on the appropriate day post induction as previously described [5].

Mice received $225 \mathrm{mg}$ of either anti-Hmgb1 antibody or the control IgY (products ST326052233, IBL International $\mathrm{GmbH}$ ), IP daily on days 2 and 3 postinduction, and sacrificed on day $4(\mathrm{n}=4$ in each cohort).

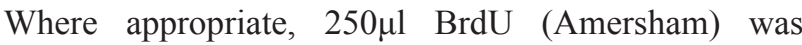
administered via IP injection $2 \mathrm{hrs}$ before sacrifice.

\section{Phenotypic characterisation of intestinal sections}

Immunohistochemistry (IHC) was performed on paraffin embedded tissues fixed in $4 \%$ formaldehyde at $4^{\circ} \mathrm{C}$ for no more than 24 hours prior to processing. The following antibodies were used for IHC: BrdU 1:500 BD biosciences; Ki671:100 Vector Labs ; Caspase 3 1:750 R\&D systems; $\beta$-catenin 1:50 C19220, Transduction Laboratories.

Crypt length from the base of a crypt to the top of the aberrant morphology displaying crypt-like features was measured along 50 clear crypt/villus axes for each sample. Apoptosis and mitotic index were scored from H\&E stained sections as previously described [5].

\section{Western analysis}

Protein was extracted (from 50-100 mg tissue) using RIPA lysis buffer and protein concentrations determined (BCA kit, Pierce). $60 \mu \mathrm{g}$ of cellular protein was separated on a $10 \%$ polyacrylamide gel and transferred to nitrocellulose (Schleicher\&Schuell). Following a blockinf step (TBS containing 5\% non fat dry milk, $2 \%$ donkey
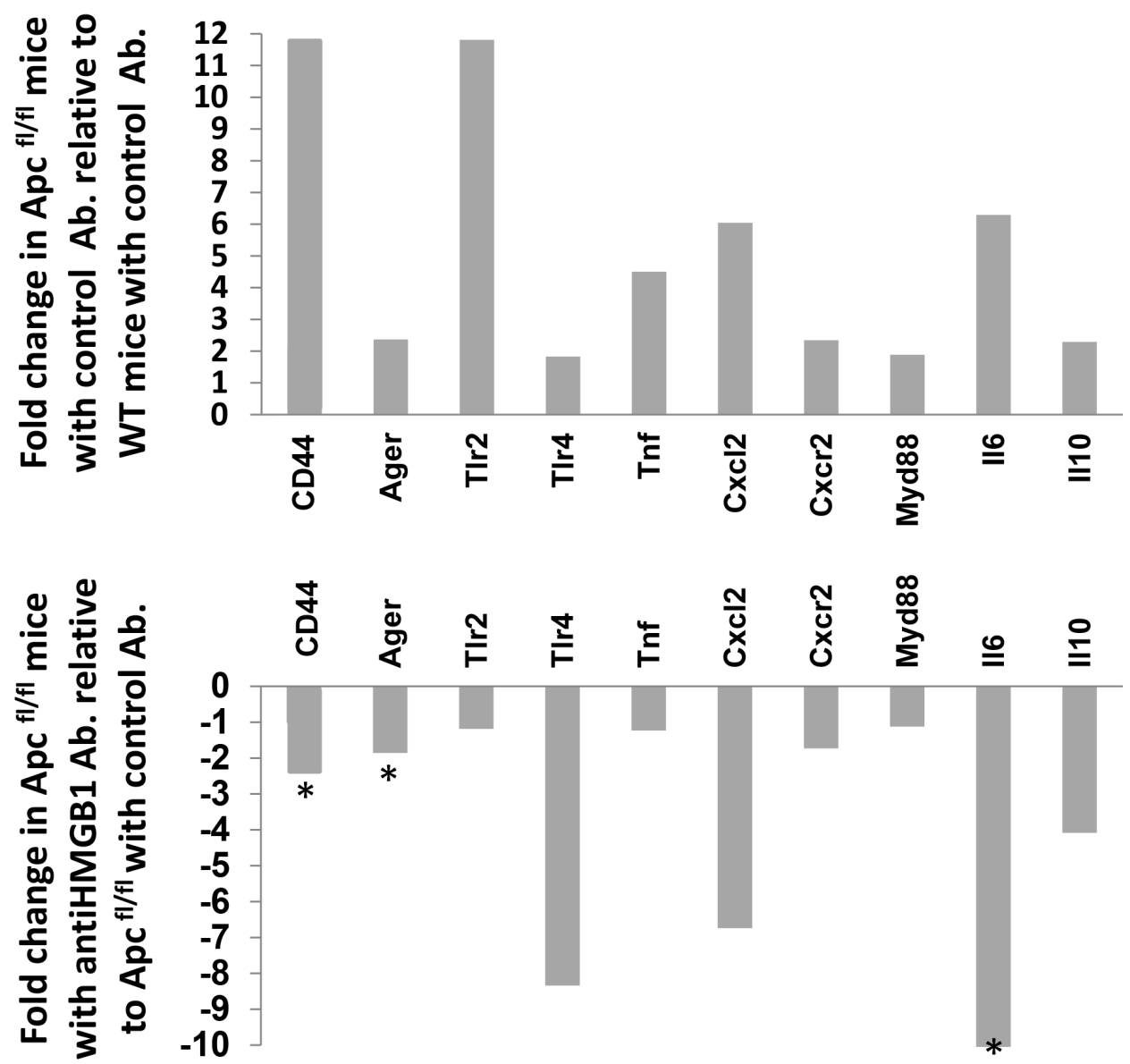

Figure 4: Expression analysis of inflammatory response genes in Wnt activated intestine following neutralising HMGB1-antibody treatment. Relative fold-change of a panel of inflammatory response genes in whole intestinal extracts following the loss of $A p c$ compared to control (top panel) and in $A p c^{f t o x}$ mice following neutralising HMGB1-antibody treatment compared to control IgY treatment. * denotes significance determined using Whitney $\mathrm{U}$ test of $\Delta \mathrm{CT}$ values at $\mathrm{P}<0.05$. 
serum, $0.02 \% \mathrm{TX}-100,0.02 \% \mathrm{NaN} 3)$ the following antibodies were used: anti-HMGB1 1:1,000 (TECAN), mouse anti-actin (ICN) 1:1,000; HRP-conjugated secondary donkey antibodies (Diagnostics Scotland) 1:5,000. SuperSignal West Femto ECL reagent (Pierce) was used for detection.

\section{qRT_PCR}

RNA extracted from whole intestinal samples, was used to synthesise first strand cDNA using a VersoTM cDNA Kit (Thermo Scientific) and anchored oligo-dT primers (Thermo Scientific) according to the manufacturer's instructions. All qRT-PCR reactions were run on the Applied Biosystems StepOnePlus machine. The programming was carried out using the computer-based StepOnePlus software. Primers were designed using the Universal ProbeLibrary Assay Design Centre (Roche).
Intra-peritoneal (IP); Immunohistochemistry (IHC); dextran sulfate sodium salt (DSS); reactive oxygen species (ROS).

\section{ACKNOWLEDGMENTS}

This work was supported by a Cancer Research UK program grant awarded to ARC (A15937), a Wellcome Trust VIP award awarded to KRR, a school of biosciences seed corn fund awarded to KRR, and a North West Cancer Research Fund awarded to JRJ. We thank Mark Bishop, Matthew Zverev and Derek Scarborough for technical services and support with genotyping and histology.

\section{CONFLICTS OF INTEREST}

The authors declare no conflicts of interest.

\begin{tabular}{lcc}
\hline Gene Name & FWD sequence & REV sequence \\
\hline beta-Actin & ctaaggccaaccgtgaaag & accagaggcatacagggaca \\
CD44 & tccttcttatccggagcac & acgtctcctgctgggtagc \\
Ager & ggtccactggataaggatgg & taggtgccctcatcctcgt \\
Tlr2 & ggggcttcacttctctgctt & agcatcctctgagatttgacg \\
Tlr4 & ggactctgatcatggcactg & ctgatccatgcattggtaggt \\
Tnf & tcttctcattcctgcttgtgg & ggtctgggccatagaactga \\
Cxcl2 & aaaatcatccaaagatactgaacaa & ctttggttcttccgttgagg \\
Cxcr2 & caggaccaggaatgggagta & tccctccaaatatcccta \\
Myd88 & tgacttccagaccaagtttgc & gaatcagtcgcttctgttgga \\
Il6 & tctaattcatatcttcaaccaagagg & tggtccttagccactccttc \\
Il10 & cagagccacatgctcctaga & gtccagctggtcctttgttt \\
Hmgb1 & ttgggtcacatggattattagtgt & cagggcatgtggacaaaag \\
\hline
\end{tabular}

\section{ELISA and MS/MS analysis}

Immediately following cervical dislocation, blood was collected directly from the chest cavity and transferred into anti-clotting tubes (Sarstedt), kept on ice for a minimal time, then centrifuged at full speed for $2 \mathrm{~min}$ to separate serum and cell pellets. Serum was transferred into fresh tubes and snap-frozen in liquid nitrogen. HMGB1 protein in serum was quantified by ELISA (Shino-Test Corp.) following the manufacturer's instructions. Mass Spectrometric characterisation of HMGB1 (MS/MS) was conducted as previously described [31-33].

\section{List of abbreviations used (if any)}

Colorectal cancer (CRC); Adenomatous polyposis coli (APC); High Mobility Group Box 1 (Hmgb1); Tandom Mass spectrometry (MS/MS); Tcf binding element (TBE);

\section{Authors' contributions}

KRR, ARC and JRJ designed the project. FS, MAY, $\mathrm{NH}$, and NPG undertook the data analysis; DJA performed the MS/MS analysis. KRR drafted the manuscript and all authors edited and approved the final version.

\section{REFERENCES}

1. Gregorieff A, Pinto D, Begthel H, Destree O, Kielman M, Clevers H. Expression pattern of Wnt signaling components in the adult intestine. Gastroenterology. 2005; 129:626-638.

2. Albuquerque C, Baltazar C, Filipe B, Penha F, Pereira T, Smits R, Cravo M, Lage P, Fidalgo P, Claro I, Rodrigues P, Veiga I, Ramos JS, Fonseca I, Leitao CN, Fodde R. Colorectal cancers show distinct mutation spectra in members of the canonical WNT signaling pathway 
according to their anatomical location and type of genetic instability. Genes Chromosomes Cancer. 2010; 49:746-759.

3. Yang Y, Yang JJ, Tao H, Jin WS. New perspectives on beta-catenin control of cell fate and proliferation in colon cancer. Food Chem Toxicol. 2014; 74:14-19.

4. Clarke AR. Wnt signalling in the mouse intestine. Oncogene. 2006; 25:7512-7521.

5. Sansom OJ, Reed KR, Hayes AJ, Ireland H, Brinkmann H, Newton IP, Batlle E, Simon-Assmann P, Clevers H, Nathke IS, Clarke AR, Winton DJ. Loss of Apc in vivo immediately perturbs Wnt signaling, differentiation, and migration. Genes Dev. 2004; 18:1385-1390.

6. Reed KR, Korobko IV, Ninkina N, Korobko EV, Hopkins BR, Platt JL, Buchman V, Clarke AR. Hunk/Mak-v is a negative regulator of intestinal cell proliferation. BMC cancer. 2015; 15:110.

7. Hammoudi A, Song F, Reed KR, Jenkins RE, Meniel VS, Watson AJ, Pritchard DM, Clarke AR, Jenkins JR. Proteomic profiling of a mouse model of acute intestinal Apc deletion leads to identification of potential novel biomarkers of human colorectal cancer (CRC). Biochemical and biophysical research communications. 2013; 440:364-370.

8. Sims GP, Rowe DC, Rietdijk ST, Herbst R, Coyle AJ. HMGB1 and RAGE in inflammation and cancer. Annual review of immunology. 2010; 28:367-388.

9. Yusein-Myashkova S, Ugrinova I, Pasheva E. Non-histone protein HMGB1 inhibits the repair of cisplatin damaged DNA in NIH-3T3 murine fibroblasts. BMB reports. 2013.

10. Nehil M, Paquette J, Tokuyasu T, McCormick F. High mobility group box 1 promotes tumor cell migration through epigenetic silencing of semaphorin $3 \mathrm{~A}$. Oncogene. 2014; 33:5151-5162.

11. Kang R, Zhang Q, Zeh HJ, 3rd, Lotze MT, Tang D. HMGB1 in cancer: good, bad, or both? Clin Cancer Res. 2013; 19:4046-4057.

12. Liu Y, Prasad R, Wilson SH. HMGB1: roles in base excision repair and related function. Biochimica et biophysica acta. 2010; 1799:119-130.

13. Merenmies J, Pihlaskari R, Laitinen J, Wartiovaara J, Rauvala H. $30-\mathrm{kDa}$ heparin-binding protein of brain (amphoterin) involved in neurite outgrowth. Amino acid sequence and localization in the filopodia of the advancing plasma membrane. J Biol Chem. 1991; 266:16722-16729.

14. Fages C, Nolo R, Huttunen HJ, Eskelinen E, Rauvala H. Regulation of cell migration by amphoterin. Journal of cell science. 2000; 113 ( Pt 4):611-620.

15. Yang H, Pellegrini L, Napolitano A, Giorgi C, Jube S, Preti A, Jennings CJ, De Marchis F, Flores EG, Larson D, Pagano I, Tanji M, Powers A, Kanodia S, Gaudino G, Pastorino S, et al. Aspirin delays mesothelioma growth by inhibiting HMGB1-mediated tumor progression. Cell death $\&$ disease. 2015; 6:e1786.
16. Andersson U, Tracey KJ. HMGB1 is a therapeutic target for sterile inflammation and infection. Annual review of immunology. 2011; 29:139-162.

17. Zimmermann K, Volkel D, Pable S, Lindner T, Kramberger F, Bahrami S, Scheiflinger F. Native versus recombinant high-mobility group B1 proteins: functional activity in vitro. Inflammation. 2004; 28:221-229.

18. Yu Y, Tang D, Kang R. Oxidative stress-mediated HMGB1 biology. Frontiers in physiology. 2015; 6:93.

19. Gardella S, Andrei C, Ferrera D, Lotti LV, Torrisi MR, Bianchi ME, Rubartelli A. The nuclear protein HMGB1 is secreted by monocytes via a non-classical, vesicle-mediated secretory pathway. EMBO reports. 2002; 3:995-1001.

20. Tang D, Billiar TR, Lotze MT. A Janus tale of two active high mobility group box 1 (HMGB1) redox states. Molecular medicine. 2012; 18:1360-1362.

21. Venereau E, Casalgrandi M, Schiraldi M, Antoine DJ, Cattaneo A, De Marchis F, Liu J, Antonelli A, Preti A, Raeli L, Shams SS, Yang H, Varani L, Andersson U, Tracey KJ, Bachi A, et al. Mutually exclusive redox forms of HMGB1 promote cell recruitment or proinflammatory cytokine release. J Exp Med. 2012; 209:1519-1528.

22. Yao X, Zhao G, Yang H, Hong X, Bie L, Liu G. Overexpression of high-mobility group box 1 correlates with tumor progression and poor prognosis in human colorectal carcinoma. J Cancer Res Clin Oncol. 2010; 136:677-684.

23. Lee H, Song M, Shin N, Shin CH, Min BS, Kim HS, Yoo JS, Kim H. Diagnostic significance of serum HMGB1 in colorectal carcinomas. PLoS One. 2012; 7:e34318.

24. Maeda S, Hikiba Y, Shibata W, Ohmae T, Yanai A, Ogura K, Yamada S, Omata M. Essential roles of high-mobility group box 1 in the development of murine colitis and colitis-associated cancer. Biochemical and biophysical research communications. 2007; 360:394-400.

25. Heijmans J, Büller NV, Hoff E, Dihal AA, van der Poll T, van Zoelen MA, Bierhaus A, Biemond I, Hardwick JC, Hommes DW, Muncan V, van den Brink GR. Rage signalling promotes intestinal tumourigenesis. Oncogene. 2013; 32:1202-1206.

26. Lum HK, Lee KL. The human HMGB1 promoter is modulated by a silencer and an enhancer-containing intron. Biochim Biophys Acta. 2001; 1520:79-84.

27. Yang H, Antoine DJ, Andersson U, Tracey KJ. The many faces of HMGB1: molecular structure-functional activity in inflammation, apoptosis, and chemotaxis. J Leukoc Biol. 2013; 93:865-873.

28. Grivennikov SI, Wang K, Mucida D, Stewart CA, Schnabl B, Jauch D, Taniguchi K, Yu GY, Osterreicher CH, Hung KE, Datz C, Feng Y, Fearon ER, Oukka M, Tessarollo L, Coppola V, et al. Adenoma-linked barrier defects and microbial products drive IL-23/IL-17-mediated tumour growth. Nature. 2012; 491:254-258. 
29. Davé SH, Tilstra JS, Matsuoka K, Li F, DeMarco RA, BeerStolz D, Sepulveda AR, Fink MP, Lotze MT, Plevy SE. Ethyl pyruvate decreases HMGB1 release and ameliorates murine colitis. J Leukoc Biol. 2009; 86:633-643.

30. Freeman J, Smith D, Latinkic B, Ewan K, Samuel L, Zollo M, Marino N, Tyas L, Jones N, Dale TC. A functional connectome: regulation of Wnt/TCF-dependent transcription by pairs of pathway activators. Mol Cancer. 2015; 14:206.

31. Huebener P, Pradere JP, Hernandez C, Gwak GY, Caviglia JM, Mu X, Loike JD, Jenkins RE, Antoine DJ, Schwabe RF. The HMGB1/RAGE axis triggers neutrophil-mediated injury amplification following necrosis. J Clin Invest. 2015; 125:539-550.

32. Ge X, Antoine DJ, Lu Y, Arriazu E, Leung TM, Klepper AL, Branch AD, Fiel MI, Nieto N. High mobility group box-1 (HMGB1) participates in the pathogenesis of alcoholic liver disease (ALD). J Biol Chem. 2014; 289:22672-22691.

33. Yang H, Lundbäck P, Ottosson L, Erlandsson-Harris H, Venereau E, Bianchi ME, Al-Abed Y, Andersson U, Tracey $\mathrm{KJ}$, Antoine DJ. Redox modification of cysteine residues regulates the cytokine activity of high mobility group box-1 (HMGB1). Mol Med. 2012; 18:250-259. 